\title{
XIII. On the method of the least squares
}

\author{
James Ivory Esq. M.A. F.R.S.
}

To cite this article: James Ivory Esq. M.A. F.R.S. (1825) XIII. On the method of the least squares, Philosophical Magazine Series 1, 65:322, 81-88, DOI: 10.1080/14786442508628396

To link to this article: http://dx.doi.org/10.1080/14786442508628396

曲 Published online: 27 Jul 2009.

Submit your article to this journal $\pi$

LII Article views: 5

Q View related articles $₫$ 


\section{PHILOSOPHICAL MAGAZINE AND JOURNAL.}

$28^{\text {th }} F E B R U A R Y 1825$. XIII. On the Method of the Least Squares. By J. Ivonx, Esq.
M.A. F.R.S.

[Continued from p. 10.]

IN the last Number of this Journal I have attempted to demonstrate the method of the least squares without having recourse to the doctrine of probabilities. It must be remembered that the demonstration, in whatever way we attempt it, must inevitably contain something vague. But the arguments that have been adduced for preferring the solution by the method of the least squares to every other, appear to be perfectly sufficient and conclusive as far as the nature of the problem will admit. If the proof by the doctrine of probabilities be more formal, it is not on that account more satisfactory; because it involves precarious suppositions which cannot possibly be rigorously exact. But in order to derive the greatest practical utility from a system of equations of condition, it becomes necessary to connect the solution of them with the rules of chance: and we now proceed to treat the problem in this view of it.

1. It is perfectly impossible to ascertain with mathematical precision the probability of the error of an observation. There is even no proof that the probability will in all cases depend precisely in the same manner upon the magnitude of the error. All that can be accomplished in this theory is to adopt the suppositions that are least likely to deviate much from the truth.

Experience shows that great errors occur less frequently than small or middling ones. If we push this principle to the utmost length, the function $\phi(e)$, which denotes the probability of the error $e$, will be greatest when $e=0$, and it will decrease as $e$ increases. Again, abstracting from errors that are affected by a constant cause, and confining our attention to such only as are irregular and fortuitous, the errors $\pm e$ will be equally probable, which requires that

Vol. 65. No. 322. Feb. 1825 . L 
$\phi(e)=\Phi(-e):$ and, as it is most reasonable to suppose that $\phi(e)$ is a continuous function, at least within certain limits, it follows that $\phi\left(e^{2}\right)$ is the proper expression of the probability of the error $e$.

All the errors being contained between certain definite limits, suppose $\pm f$; the function $\phi\left(e^{2}\right)$ will be evanescent when $e$ is equal to or greater than $\pm f$. Therefore, strictly speaking, $p\left(e^{2}\right)$ must be a discontinuous function, having real values only between the limits $\pm f$, and no values at all beyond the same limits: but this condition will be sufficiently fulfilled if $\phi\left(e^{2}\right)$ be evanescent when $e^{2}$ is infinitely great, and have a very small value when $e^{2}=f^{2}$.

The function $\Phi\left(e^{2}\right)$ may be regarded as the ordinate of a curve, corresponding to the abscissæ $\pm e$; and the small area $d e \Phi\left(e^{2}\right)$ will denote the probability of an error between the limits $e$ and $e+d e$. Wherefore the integral $\int d e \phi\left(e^{2}\right)$, taken from $e=a$ to $e=b$, will express the probability of an error between the limits $a$ and $b$. And because all possible errors are contained between $\pm f$, or $\pm \sim$; it follows that $\int d e \varphi\left(e^{2}\right)$, between the same limits, must be equal to unit, the expression of certainty.

These are properties which it is essential that the function expressing the probability of an error must possess. Let us next consider the probability of the simultaneous existence of a number of errors.

2. The several errors $e, e^{\prime}, e^{\prime \prime}, \& c$., are independent of one another, since they arise from separate observations; their respective probabilities are, $\phi\left(e^{2}\right), \phi\left(e^{\prime 2}\right), \phi\left(e^{\prime \prime q}\right), \& c_{\text {. }}$; wherefore, by the known rules, the probability of their simultaneous existence is the product,

$$
\Phi\left(e^{2}\right) \cdot \Phi\left(e^{\prime 2}\right) \cdot \Phi\left(e^{\prime \prime 2}\right) \& \mathrm{c} .
$$

Now as every factor is always positive, and is evanescent when the error is equal to $\pm \sim$; it follows that the product will have a maximum relatively to every error, and likewise an absolute maximum for certain definite values of all the errors. If the errors be functions of $x, y, \& c$., the equations of the several maxima will be,

$$
\begin{aligned}
& \frac{1}{\varphi\left(e^{2}\right)} \cdot \frac{d \varphi\left(e^{2}\right)}{d e^{2}} \cdot \frac{d e^{2}}{d x}+\frac{1}{\varphi\left(e^{\prime y}\right)} \cdot \frac{d \varphi\left(e^{\prime 2}\right)}{d e^{\prime 2}} \cdot \frac{d e^{\prime} z}{d x}+\& \mathrm{c}_{0}=0, \\
& \frac{1}{\varphi\left(e^{2}\right)} \cdot \frac{\left.d \varphi e^{2}\right)}{d e^{2}} \cdot \frac{d e^{2}}{d y}+\frac{1}{\varphi\left(e^{\prime z}\right)} \cdot \frac{d \varphi\left(e^{\prime z}\right)}{d e^{\prime z}} \cdot \frac{d e^{\prime z}}{d y}+\& \mathrm{c}_{0}=0,
\end{aligned}
$$

and all these equations together will determine the values of $x, y, \& c$. which correspond to the absolute maximum.

Again, let $\psi$ denote a rational and integral function of $e^{2}$, $e^{\prime 2}, e^{112}, \& c$. , consisting of positive terms only. 'The function 
will therefore be always positive, and it will become infinitely great whenever any of the errors is equal to $\pm \omega$. There will therefore be a minimum relatively to every error, and an absolute minimum for certain definite values of all the errors. The equations of the several minima are respectively,

$$
\begin{aligned}
& \frac{d \psi}{d e^{2}} \cdot \frac{d e^{2}}{d x}+\frac{d \psi}{d e^{\prime 2}} \cdot \frac{d e^{\prime z}}{d x}+\& c_{.}=0, \\
& \frac{d \psi}{d e^{2}} \cdot \frac{d e^{2}}{d y}+\frac{d \psi}{d e^{\prime z}} \cdot \frac{d e^{\prime 2}}{d y}+8 c_{.}=0,
\end{aligned}
$$

and all these equations together determine the particular values of $x, y, 8$ c., in the case of the absolute minimum.

Let us now suppose that the most probable values of $\psi$ are the several minima, and consequently that the absolute minimum is the most advantageous, or the most probable, value of all: then, since the probability of $\psi$ is just the same as that of the simultaneous existence of the errors which enter into it, that probability will have the product $(P)$ for its expression. Wherefore, in the supposition we have made, it follows that the minima of one function will take place at the same time with the maxima of the other; and hence we get these formulæ, viz.

$$
\begin{aligned}
& \frac{1}{\phi\left(e^{2}\right)} \cdot \frac{d \cdot \phi\left(e^{2}\right)}{d e^{q}}=h^{2} \cdot \frac{d \psi}{d e^{2}}, \\
& \frac{1}{\phi\left(e^{\prime 2}\right)} \cdot \frac{d \cdot \phi\left(e^{\prime 2}\right)}{d e^{\prime 2}}=h^{2} \cdot \frac{d \psi}{d e^{\prime 2}},
\end{aligned}
$$

\&c.

which render the equations (B) and (C) identical, all the latter being first multiplied by the arbitrary quantity $h^{2}$. Now it is manifest that these formulæ cannot be satisfied unless $\psi$ have this form, viz.

$$
\psi=f\left(e^{2}\right)+f\left(e^{\prime 2}\right)+f\left(e^{\prime 2}\right)+8 \mathrm{c} . ;
$$

in which case all the formulæ are contained in one, viz.

$$
\frac{1}{\varphi\left(e^{2}\right)} \cdot \frac{d \varphi\left(e^{2}\right)}{d e^{2}}=h^{2} \cdot \frac{d f\left(e^{2}\right)}{d e^{2}}
$$

which determines the function $\varphi$.

If the probabilities of the several errors $e, e^{\prime}, e^{\prime \prime}, 8 \mathrm{cc}$. be expressed by different functions, viz. $\phi\left(e^{2}\right), \phi^{\prime}\left(e^{\prime 2}\right), \phi^{\prime \prime}\left(e^{\prime 2}\right), \&$ c., it will follow that $\psi$ must have this form, viz.

$$
\psi=f\left(e^{2}\right)+f^{\prime}\left(e^{\prime 2}\right)+f^{\prime \prime}\left(e^{\prime \prime 2}\right)+\& \mathrm{c} \text {; }
$$

and then the several formulæ will determine the functions $\phi, \phi^{\prime}, \phi^{\prime \prime}, \& \mathrm{c}$.

3. In order to apply the foregoing reasoning to a system of equations of condition, we must recollect that the most ad- 
vantageous, or the most probable, solution is when the sum of the squares of the errors is a minimum. Hence

consequently

$$
\begin{aligned}
& \psi=e^{2}+e^{\prime 2}+e^{1 / 2}+\& c_{.} ; \\
& \frac{1}{\phi\left(e^{2}\right)} \cdot \frac{d \phi\left(e^{2}\right)}{d e^{2}}=-h^{2}-\frac{d \psi}{d e^{2}}=-h^{2}:
\end{aligned}
$$

$$
\phi\left(e^{2}\right)=k c^{-k^{2} e^{2}},
$$

$c$ being the base of the hyperbolic logarithms. It has been shown that the integral

$$
\int d e \Phi\left(e^{2}\right)=k \int d e c^{-h^{2} e^{2}}
$$

taken between the limits $\pm \sim$, must be equal to unit: and hence

wherefore

$$
k \int d e c^{-h^{2} e^{2}}=\frac{k}{h} \cdot \sqrt{\pi}=1 ;
$$

$$
\begin{gathered}
k=\frac{h}{\sqrt{\pi}}, \text { and } \\
\phi\left(e^{2}\right)=\frac{h}{\sqrt{\pi}} c^{-h^{2} e^{2}} .
\end{gathered}
$$

In order to determine $h$ we must employ another consideration. The integral

$$
\int e^{2} d e \cdot \varphi\left(e^{2}\right)=\frac{h}{\sqrt{x}} \int e^{2} d e c^{-h^{2} e^{2}},
$$

taken between the limits $\pm \sim$, is equal to the sum of the squares of the errors multiplied by their respective probabilities; and it is therefore the limit to which the mean of the sum of the squares of the errors converges as the number of the observations increases. Now the integral is equal to $\frac{1}{2 h^{2}}$; and, if we denote by $\varepsilon, \varepsilon^{\prime}, \varepsilon^{\prime \prime}, \&$ c., the errors of the most advantageous solution, or those of which the sum of the squares is a minimum, we shall have very nearly when the number of observations is great

$$
\frac{1}{2 k^{2}}=\frac{\varepsilon^{2}+\varepsilon^{\prime 2}+\epsilon^{\prime \prime} q+\& c .}{n}
$$

$n$ being the number of the errors. Hence, employing the summatorial prefix $S$, we get,

$$
h=\sqrt{\frac{x}{2 \mathrm{~S} \cdot \varepsilon^{2}}} .
$$

Thus every thing is known -in the function expressing the probability of an error. The quantity $h$ may be considered as measuring the precision of the observations. For $\frac{1}{h^{2}}$ is proportional to $\frac{\mathbf{S . s ^ { 2 }}}{n}$; and as the latter quantity is independent 
of $n$ when the number of the observations is great, it follows that $\frac{1}{h^{2}}$ will be greater or less according as the errors, taken upon the whole, are more or less considerable; that is, according as the observations are less or more exact.

4. Let us now consider this system of equations of condition, viz.

$$
\begin{aligned}
& e=a x+b y+c z-m \\
& e^{\prime}=a^{\prime} x+b^{\prime} y+c^{\prime} z-m^{\prime} \\
& e^{\prime \prime}=a^{\prime \prime} x+b^{\prime \prime} y+c^{\prime \prime} z-m^{\prime \prime} \\
& \quad \text { \&c. }
\end{aligned}
$$

the quantities $x, y, z$, as well as $e, e^{\prime}, e^{\prime \prime}, \& c$., being indeterminate. Put $\varepsilon, \varepsilon^{\prime}, \varepsilon^{\prime \prime}, \& \mathrm{c}$. for the particular values of $e, e^{\prime}, e^{\prime \prime}, \& \mathrm{c}$. in the most advantageous solution, or when the sum of the squares is a minimum; and let $A, B, C$ be the corresponding values of $x, y, z$, we shall have

$$
\begin{aligned}
& \varepsilon=a \mathrm{~A}+b \mathrm{~B}+c \mathrm{C}-m \\
& \varepsilon^{\prime}=a^{\prime} \mathrm{A}+b^{\prime} \mathrm{B}+c^{\prime} \mathrm{C}-m^{\prime} \\
& \varepsilon^{\prime \prime}=a^{\prime \prime} \mathrm{A}+b^{\prime} \mathrm{B}+c^{\prime \prime} \mathrm{C}-m^{\prime \prime} \\
& \& \mathrm{c} .
\end{aligned}
$$

and $\mathrm{A}, \mathrm{B}, \mathrm{C}$ will be found from the equations of the minima, viz.

$$
\begin{aligned}
& a \varepsilon+a^{\prime} \varepsilon^{\prime}+a^{\prime \prime} \varepsilon^{\prime \prime}+\& \mathrm{c} .=0 \\
& b \varepsilon+b^{\prime} \varepsilon^{\prime}+b^{\prime \prime} \varepsilon^{\prime \prime}+8 \mathrm{cc}=0 \\
& c \varepsilon+c^{\prime} \varepsilon^{\prime}+c^{\prime \prime} \varepsilon^{\prime \prime}+8 \mathrm{c} .=0 .
\end{aligned}
$$

Again, let

$$
\begin{aligned}
& x=\mathrm{A}+u \\
& y=\mathrm{B}+v \\
& z=\mathrm{C}+w
\end{aligned}
$$

and we may regard $\psi, v, w$ as the respective errors of $\mathrm{A}, \mathrm{B}, \mathrm{C}$. Substitute now the expressions of $x, y, z$, in the values of $e$, $e^{\prime}, e^{\prime \prime}, \& c .$, and we shall have

$$
\begin{aligned}
& e=\varepsilon+a u+b v+c w \\
& e^{\prime}=\varepsilon^{\prime}+a^{\prime} u+b^{\prime} v+c^{\prime} w \\
& e^{\prime}=\varepsilon^{\prime \prime}+a^{\prime \prime} u+b^{\prime \prime} v+c^{\prime \prime} w \\
& \& c .
\end{aligned}
$$

Further, put $\lambda=(a u+b v+c w)^{3}$

$$
\begin{aligned}
& +\left(a^{\prime} u+b^{\prime} v+c^{\prime} w\right)^{2} \\
& +\left(a^{\prime \prime} u+b^{\prime \prime} v+c^{\prime \prime} w\right)^{8} \\
& +\& c .
\end{aligned}
$$

then square the values of $e, e^{\prime}, e^{\prime \prime}, \& \mathrm{c}$; add the results into one sum; and leave out the terms which, on account of the equations of the minima, are equal to zero; we shall get

$$
\mathrm{S} . e^{2}=\mathrm{S} . \varepsilon^{2}+\lambda \text {. }
$$

Now the probability of the function $S_{0} e^{2}$ is equal to that of the simultaneous existence of the errors whose squares are 
added together: it is therefore proportional to the product (P), or to the exponential quantity

$$
c^{-h^{2} \text { S. } e^{2}}=c^{-h^{2} \text { S. } \iota^{2}-h^{2} \lambda} \text {. }
$$

Wherefore leaving out the constant factor, the probability of S. $e^{2}$, or, which is the same thing, the probability of the simultaneous existence of the errors $u, v, r$, is proportional to

$$
c^{-k^{a} \lambda} \text {. }
$$

In order to find the separate probability of the error $u$, we must take the sum of all the values of the foregoing expression that arise by combining $u$ with every possible value of $v$ and $w:$ it is therefore proportional to the fluent,

$$
\int d v d w c^{-h^{2} \lambda}
$$

both the integrations being executed between the limit $\pm \sim$.

To determine the integral, expand the squares in the value of $\lambda$, and collect the like terms; then

$$
\begin{aligned}
\lambda & =u^{2} \mathrm{~S} \cdot a^{2}+v^{2} \mathrm{~S} \cdot b^{2}+w^{2} \mathrm{~S} \cdot c^{2} \\
& +2 u v \mathrm{~S} \cdot a b+2 u w \mathrm{~S} \cdot a c+2 v w \mathrm{~S} \cdot b c .
\end{aligned}
$$

Again, assume $t=\mathrm{P} u$,

$$
\begin{aligned}
& t^{\prime}=\mathbf{P}^{\prime} u+\mathbf{Q} v, \\
& t^{\prime \prime}=\mathbf{P}^{\prime \prime} u+\mathbf{Q}^{\prime} v+\mathbf{R} w,
\end{aligned}
$$

and determine the arbitrary coefficients so as to satisfy the condition

$$
\lambda=t^{2}+t^{\prime 2}+t^{\prime \prime 2} \text {. }
$$

By equating the coefficients of the like terms of this equation, we shall get

$$
\begin{array}{rr}
\mathbf{P}^{2}+\mathbf{P}^{\prime 2}+\mathbf{P}^{\prime \prime 2}=\mathrm{S} . a^{2}, & \mathbf{P}^{\prime} \mathbf{Q}+\mathrm{P}^{\prime \prime} \mathbf{Q}^{\prime}=\mathbf{S} . a b \\
\mathbf{Q}^{2}+\mathbf{Q}^{\prime 2}=\mathrm{S} . b^{2}, & \mathbf{P}^{\prime \prime} \mathbf{R}=\mathrm{S} . a c \\
\mathbf{R}^{2}=\mathrm{S} . c^{2}, & \mathbf{Q}^{\prime} \mathbf{R}=\mathrm{S} . b c .
\end{array}
$$

Hence we obtain $P^{z}=\frac{M}{N}$; the values of $M$ and $N$ being as follows, viz.

$$
\begin{aligned}
& \mathrm{M}=\mathrm{S} . a^{2} \times \mathrm{S} . b^{2} \times \mathrm{S} . c^{2}+2 \mathrm{~S} . a b \times \mathrm{S} . a c \times \mathrm{S} . b c \\
&-\mathrm{S} . a^{2} \times(\mathrm{S} . b c)^{2}-\mathrm{S} . b^{2} \times(\mathrm{S} . a c)^{2}-\mathrm{S} . c^{2} \times(\mathrm{S} . a b) \\
& \mathrm{N}=\mathrm{S} . b^{2} \times \mathrm{S} . c^{2}-(\mathrm{S} . b c)^{2}:
\end{aligned}
$$

and it is easy to prove that $M$ and $N$ will be always positive. It will not be necessary to determine the other coefficients. We shall now have

$$
\int d v d w c^{-h^{2} \lambda}=\frac{1}{\mathrm{QR}} \cdot \int d t^{\prime} d t^{\prime \prime} c^{-h^{2} t^{2}-h^{2} t^{\prime 2}-h^{2} t^{\prime \prime 2}},
$$

the limits of the integrations being the same as before. Wherefore the probability of the error $u$ is proportional to

$$
\frac{c^{-h^{2} t^{2}}}{\mathbf{Q R}} \times \int d t^{\prime} c^{-h^{2} t^{\prime 2}} \times \int d t^{\prime \prime} c^{-h^{2} t^{\prime \prime 2}} ; \quad \text { and }
$$


and as the integrations produce constant quantities only, the same probability will be equal to the expression,

$$
k c^{-h^{2} t^{2}}=k c^{-h^{2} \mathbf{P}^{2} u^{2}} \text {. }
$$

The constant $k$ will be determined by observing that the integral

$$
k \int d u c^{-h^{2} \mathbf{P}^{2} u^{2}}
$$

taken between the limits $\pm \omega$, comprehends every possible error, and it must therefore be equal to unit. Hence $\frac{k \sqrt{\pi}}{h \mathrm{P}}$ $=1$; and $k=\frac{h \mathrm{P}}{\sqrt{\pi}}$. Wherefore, finally, the probability of the error $u$, in the value $\mathrm{A}$ found by the method of the least squares, is equal to

$$
\frac{h \mathrm{P}}{\sqrt{x}} c^{-h^{2} \mathrm{P}^{2} u^{2}}
$$

If we compare this expression with the error of an original observation, it will appear that the precision of $A$, the value of the element found by the method of the least squares, is to the precision of the actual observations, as $h \mathrm{P}$ to $h$, or as $P$ to 1 . The probability that the true value of the element is between the limits $A(1 \pm \delta)$ is equal to the integral

taken between the limits $\pm A \delta$.

$$
\frac{h \mathbf{P}}{\sqrt{ } \approx} \int d u c^{-h^{3} \mathbf{P}^{2} u^{2}}
$$

It is easy to transfer what has been proved with respect to $u$, the error of $\mathrm{A}$, to $v$ and $w$, the errors of $\mathrm{B}$ and $\mathrm{C}$. As the solution we have given extends to three elements, it will necessarily comprehend the subordinate cases of one and two elements; and there is no difficulty, except the length of the operations, of applying the same analysis to any number of elements.

It is not my intention to treat of the practical details of this Theory, but merely to lay before the reader that particular view of its principles which appears most natural and philosophical. All that part connected with the doctrine of chance, is founded on the hypothesis that in all cases the probability of an error depends precisely in the same way on the magnitude of the error, or that it is always the same function of the error. Now, I believe, it will be allowed that the grounds of this supposition are much less sure than the evidence adduced in proof of the method of the least squares. There would therefore be a greal logical fault in making the most advantageous solution of a system of equations of con- 
dition depend upon the arbitrary expression of the probability of an error. But when we set out with demonstrating the most advantageous solution from the nature of the equations of conditions, the whole theory follows naturally, and is placed on its proper foundation.

For better illustrating the principles of this important speculation, I shall resume the subject on a future occasion, and offer some further remarks upon it.

February 3, 1825.

JAMES Ivohy.

\section{On the Marmolite of Mr. NutTall. By Lardner VANUXEM*.}

THE description and analysis of this mineral was published by Mr. Thomas Nuttall, in vol. iv. No. 1. of the American Journal of Science and Arts.

Having last summer visited with Professor Keating the Hoboken locality of serpentine $t, I$ was enabled to make a number of observations; the communication of which I hope will not be uninteresting, or considered unimportant by the Academy.

For some years past considerable doubt has prevailed among many of the best mineralogists with respect to the propriety of retaining serpentine as a mineral species, having few or none of those external characters required to substantiate its claim to such a rank. By some, serpentine has been considered as a rock, whose substance appeared to be the result rather of a mixture of different minerals, or the elements of different minerals, melted and deposited in a confused state, than an homogeneous substance or simple mineral whose aggregate has been effected by the power of chemical affinity. That it is not generally regarded to be a mineral, sui generis,

- From Jour. of the Acad. of Nat. Sciences of Philadelphia, vol. iii. p. 129.

+ The author of the paper on the marmolite considers the serpentine of Hoboken, (N.J.) to "appertain rather to the transition than the primitive range;" on what ground 1 know not, further than the circumstance of a part of it being in fragments, and these fragments connected together so as to form a breccia: but this fact is susceptible of an explanation by which the primordial character of the mass remains in all its integrity. Serpentine, like many other rocks, is split or cracked in various directions; in some parts of the serpentine of Hoboken, the cracks or fissures are very numerous, of caurse the fragments are small. These fissures in many instances are filled with carbonate of lime, so that the fragments form one solid mass. Now this fact is analogous to those parts of a rock, one of the primitive class for example, which are traversed in different directions by veins in series; and no one ever supposed that the intervening masses lost their primitive character from the presence of such veins. 